\title{
A Clinical and Histological Study of Radiofrequency-Assisted Liposuction (RFAL) Mediated Skin Tightening and Cellulite Improvement
}

\author{
_-RFAL for Skin Tightening
}

\section{Marc Divaris $^{1}$, Sylvie Boisnic ${ }^{2}$, Marie-Christine Branchet ${ }^{2}$, Malcolm D. Paul ${ }^{3}$}

${ }^{1}$ Plastic and Maxillo-Facial Surgery, University of Pitie Salpetiere, Paris, France; ${ }^{2}$ Institution GREDECO, Paris, France; ${ }^{3}$ Department of Surgery, Aesthetic and PlasticSurgery Institute, University of California, Irvine, USA.

Email:mdivaris@wanadoo.fr

Received May $1^{\text {st }}, 2011$; revised May 27 ${ }^{\text {th }}, 2011$; accepted June $6^{\text {th }}, 2011$.

\begin{abstract}
Background: A novel Radiofrequency-Assisted Liposuction (RFAL) technology was evaluated clinically. Parallel original histological studies were conducted to substantiate the technology's efficacy in skin tightening, and cellulite improvement. Methods: BodyTite ${ }^{T M}$ system, utilizing the RFAL technology, was used for treating patients on abdomen, hips, flanks and arms. Clinical results were measured on 53 patients up to 6 months follow-up. Histological and biochemical studies were conducted on 10 donors by using a unique GREDECO model of skin fragments cultured under survival conditions. Fragments from RFAL treated and control areas were examined immediately and after 10 days in culture, representing long-term results. Skin fragments from patients with cellulite were also examined. Results: Gradual improvement in circumference reduction $(3.9-4.9 \mathrm{~cm})$ and linear contraction $(8 \%-38 \%)$ was observed until the third month. These results stabilized at 6 months. No adverse events were recorded. Results were graded as excellent by most patients, including the satisfaction from minimal pain, bleeding, and downtime. Histological analysis of skin fragments immediately following RFAL treatment showed hypodermal coagulation of fat and blood vessels, and structural changes in dermal fibers. After 20 days in culture, fibroblast fibers metabolism was stimulated. Histological changes following cellulite treatment were also noted. Conclusions: The novel RFAL technology offers immediate skin tightening effect that is stable over time. The histological results correlated with the clinical observations of circumference reduction and skin tightening, as expressed by tissue fibers modulation. Moreover, RFAL offers an effective treatment for cellulite by formation of a thick collagen band at the dermal-hypodermal junction.
\end{abstract}

Keywords: Radiofrequency, Skin Tightening, Cellulite

\section{Introduction}

Traditional liposuction often results in excessive loose skin [1]. The addition of internal energy, such as ultrasound $[2,3]$ resulted in marginal improvement in skin contraction. Laser-assisted liposuction [4,5] demonstrated better tightening capabilities, but only in small body areas. The ability to apply heat with liposuction in large body areas and to improve skin tightening dramatically has been demonstrated by the RadiofrequencyAssisted Liposuction (RFAL), applied by the BodyTite ${ }^{\mathrm{TM}}$ system (Invasix Ltd., Yokneam, Israel) [6-9].

It is well established that radiofrequency (RF) induces new collagen and elastin synthesis [10]. In this study we analyzed the tightening effect after treatment by RFAL, using a novel histological method for evaluation of collagen and elastic fibers modification in the dermis (GREDECO, Paris, France). The effect of RFAL on cellulite was also tested.

The aim of this study was to analyze the effect of RFAL in in-vivo and ex-vivo models and to match the clinical results with the histological and biochemical re- 
sults.

\section{Materials and Methods}

\subsection{System}

The BodyTite ${ }^{\mathrm{TM}}$ system has a hand piece composed of internal and external electrodes [6,9]. The internal electrode is a cannula, which permits RF and heat emission simultaneously with fat aspiration. The controllable settings of the system include RF power, cut-off temperature and high and low impedance limits.

\subsection{Clinical Study Design}

The procedure was performed on 53 patients (42 women and 11 men), conducted at the Spontini Aesthetic Clinic in Paris, France. The treated body sites were 42 abdomens, flanks and hips and 14 arms. Cellulite samples were taken from buttocks and thighs.

The clinical protocol started with classic suction-assisted liposuction (SAL), followed by RFAL for skin tightening, yielding a fast procedure. It is, however, possible to start with RFAL and complete the aspiration with SAL, or perform the whole procedure with RFAL.

\subsection{Clinical Procedure}

The clinical protocol followed super-wet tumescent fluid infiltration according to the Klein technique [11]. The settings used were 30 - $40 \mathrm{~W}$ for arms, and 55 - $65 \mathrm{~W}$ for abdomen, hips and flanks. Cut-off temperature settings were $38^{\circ} \mathrm{C}-41^{\circ} \mathrm{C}$, maintained for $\sim 2$ minutes until the desired effect was reached. Treatment areas were divided to $\sim 12 \times 12 \mathrm{~cm}$ thermal zones and the mean effective energy delivered to each treated zone was $\sim 10 \mathrm{~kJ}$. The whole procedure took 4 - 5 minutes/thermal zone.

The arms were treated on posterior and interior parts to correct the loose and wrinkly appearance, respectively. While treating the abdominal region, the patient was lying in both supine and prone positions in order to treat the full circumference of the abdomen, hips and flanks.

\subsection{In-Vivo Results Analysis}

Results of circumference measurements were analyzed pre-treatment, and then immediately, 6 weeks, 3 months and 6 months post-treatment.

Six women with pronounced cellulite on thighs and buttocks were followed to record the changes 3 months post treatment.

\subsection{Ex-Vivo Study}

The GREDECO technology [12-14], is an ex-vivo model that enables the survival of full thickness skin fragments up to 21 days. Using this model, no histological, immu- nological, and biochemical changes were recorded when compared to in-vivo state. The metabolic rate was found to be four times faster than in-vivo [15].

Skin fragments were recovered after abdominoplasty from 10 donors of the clinical study participants, following RFAL treatment and were tested versus untreated control areas. One portion of each fragment was fixed immediately after treatment (D0) in formol and was embedded in paraffin for histological analysis. Another portion of each fragment was cultured for 10 days (D10) in the GREDECO ex-vivo model and was used to analyze fibers synthesis. Each skin sample was placed on a porous membrane and positioned in a culture well with medium, kept in a sterilizer at $37^{\circ} \mathrm{C}$ and in air/ $\mathrm{CO}_{2}(95 \% / 5 \%)$.

Serial sections of $4 \mu \mathrm{m}$ thicknesses were obtained from untreated and treated fragments at D0 or D10.

\subsection{Ex-Vivo Results Analysis}

Histological evaluation of skin fragments at D0: sections stained with hemalun-eosin were compared for size and appearance of fat cells and coagulation of hypodermal blood vessels.

Morphometric analysis of collagen fibers at D10: sections were stained with a collagen specific staining of picric acid containing $0.1 \%$ Sirius-red. Collagen was analyzed by computerized morphometric image analysis. The stained slides were examined microscopically and photographed. The surface of collagen bundles was measured in $\mu^{2}$ and the relative collagen content of the dermis was expressed as percentage of surface of analyzed dermis.

Morphometric analysis of elastic fibers at D10: slides stained by elastin specific staining of $(+)$ catechine were analyzed by computerized morphometric image analysis. Elastic fibers from superficial and mid dermis were counted as percentage of surface of analyzed dermis. The length of elastic fibers was also measured.

Collagen synthesis at D10: skin fragments were enzymatically digested overnight at $4^{\circ} \mathrm{C}$, in $0.5 \mathrm{M}$ acetic acid solution, containing pepsine. The fibroblastic activity of collagen synthesis was evaluated by a spectrocolorimetric method $(540 \mathrm{~nm})$, measuring the acido-soluble new collagen synthetized after specific staining by Sirius red (Sircoll Collagen Assay, Interchim). Results were expressed in $\mu$ g collagen/mg skin biopsy.

Elastin synthesis at D10: the fibroblastic activity for elastin synthesis was evaluated in supernatants by a spectrocolorimetric method (513 nm), measuring the tropoelastin synthesized after a specific staining by elastin-dye complex (Sircoll Elastin Assay, Interchim). Results were expressed in $\mu$ g elastin $/ \mathrm{ml}$. 


\subsection{Statistical Analysis}

Mean values and standard deviations were calculated for quantitative variables. The statistical significance of changes recorded was determined with the Student's t-test, whereby $\mathrm{p}<0.05$ is considered statistically significant.

\section{Results}

\subsection{Clinical Study}

All 53 patients were treated with suction-assisted liposuction (SAL) followed by RFAL. Following treatment, we observed an instantaneous firming of the skin and underlying tissue, ranging between $6 \%-9 \%$ linear contraction. Skin contraction quantification was done by measurements in 2 and 3 dimensions.

2-dimensions: measurements of the distance between 2 points before and after treatment indicated the percent of linear contraction. Cutaneous shrinkage of arms was remarkable, with a linear contraction that increased up to 3 months and stabilized at 6 month post treatment (Figure 1). Abdomen results were more dramatic and followed a similar pattern (Figure 2). All patients showed abdominal and arms skin tightening following RFAL treatment that was statistically significant $(p<0.05)$. Average linear contraction at 6 months follow-up was $19.6 \%$ for arms and
$25.2 \%$ for abdomen with extreme values of $8 \%$ - 38\%.

3-dimensions: Arms and abdomen circumference reduction was noted (Figures 3-5) and showed a similar time-response pattern to the linear contraction. Representative photos of arms (Figures 3 and 4) and abdomen (Figure 5) are shown. Average circumference reduction at 6 months follow-up was $3.8 \mathrm{~cm}$ for arms and $4.7 \mathrm{~cm}$ for abdomen. Third dimensional shrinkage in skin depth was also observed when comparing abdominal RFALtreated zones to control. Cellulite on thighs or buttocks showed a significant improvement 3 months post treatment (Figure 6). In addition, a reduction in ecchymosis and edema was observed, (in accordance with other study) [7], provoking less patient discomfort and quicker recuperation. Patient satisfaction at 6 months time was always positive except 1 case out of 23 arm cases that had excessive loose skin. No adverse events were recorded in all patients throughout the study.

\subsection{Histological Study}

Immediate Results: skin analysis of all abdominal fragments immediately after RFAL treatment showed coagulation of blood vessels and fat near the tract of the internal electrode (Figure 7). All donors showed significant immediate modification of collagen fibers, expressed as a

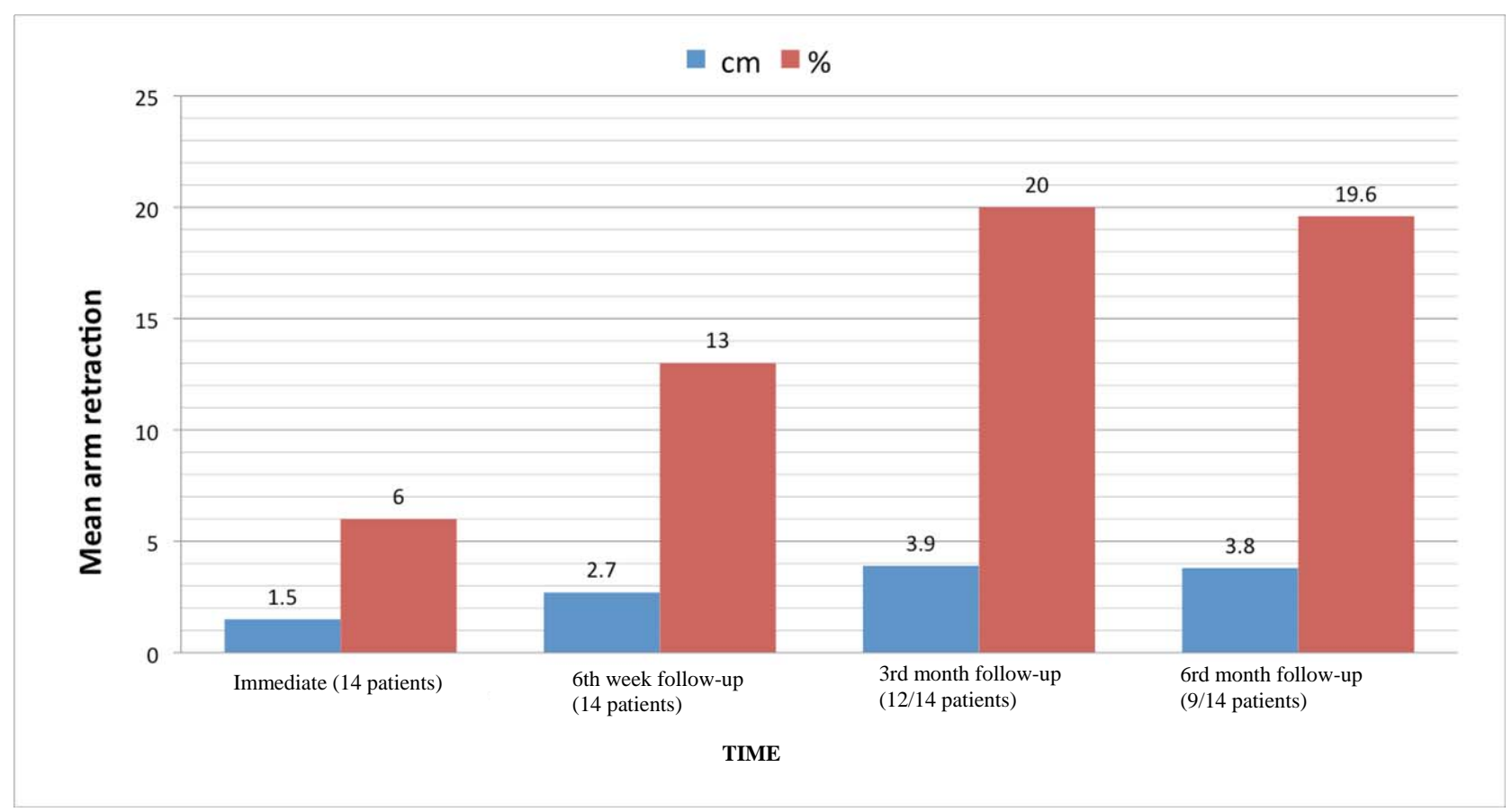

Figure 1. Diagram of time dependent mean arm retraction, expressing linear contraction (\%), and circumference reduction (cm). 


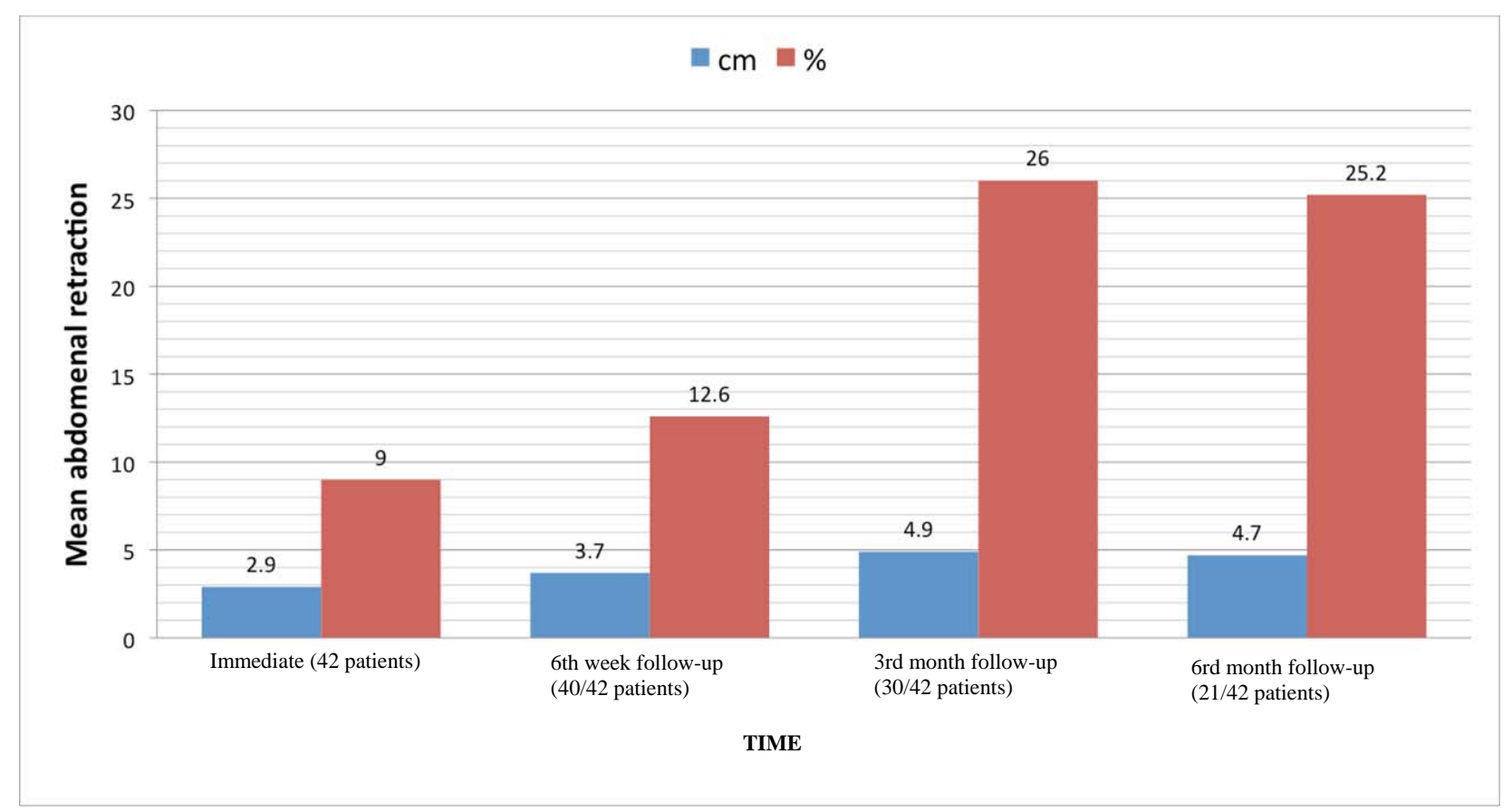

Figure 2. Diagram of time dependant mean abdominal retraction, expressing linear contraction (\%), and circumference reduction (cm).

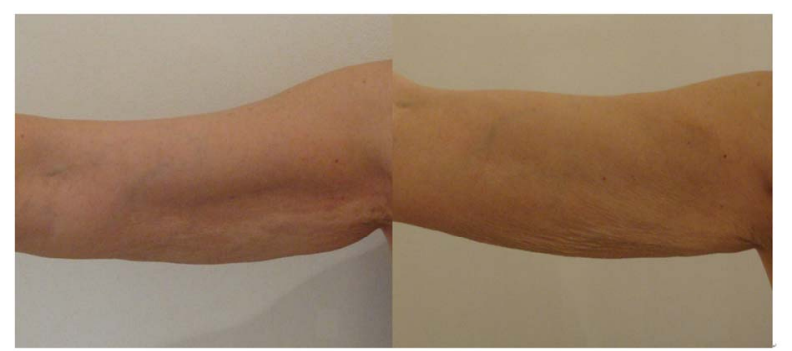

(a)

(b)

Figure 3. Woman's (58 years old) arm with loose skin without fat (a) and post-operational result after 1 month with good shrinkage of loose skin aspect (b).

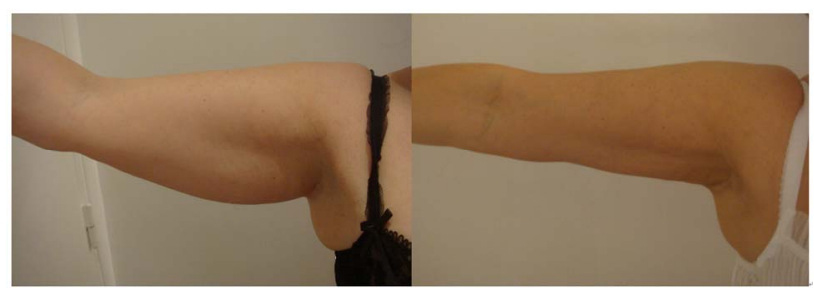

(a)

(b)

Figure 4. Woman's (49 years old) arm with fat (a), and post-operational result after 3 months with a reduction of $4.5 \mathrm{~cm}$ (b).

decrease of space between collagen fibers in the superficial and mid dermis (Figure 8). These results correlate with the immediate skin tightening in-vivo.

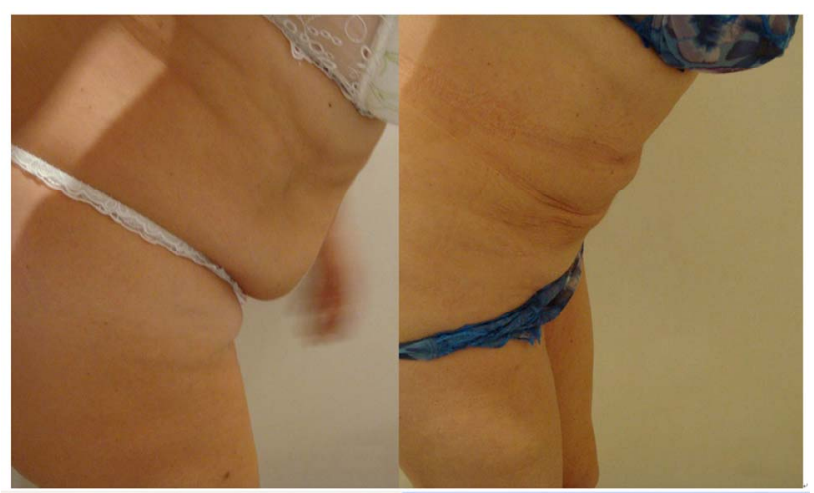

(a)

(b)

Figure 5. Woman (54 years old) in "en position antefléchie” (a), and post-operational results after 6 months with significant improvement of abdomen and a circumference reduction of $6.5 \mathrm{~cm} \mathrm{(b)}$.

A destruction of collagen fibers was observed in the deep dermis and at the dermal-hypodermal junction after treatment, manifested as thinning of spaced collagen bundles (Figure 9). This may indicate the capacity for collagen remodeling, and is in correlation with the long-term clinical findings. Modifications of elastic fibers after the treatment were observed at the dermalhypodermal-junction, showing partial destruction of elastic fibers. This was not seen in the superficial and mid dermis. 


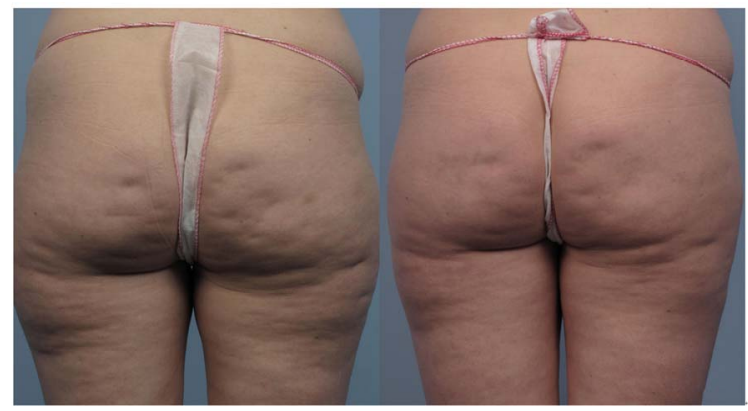

(a)

(b)

Figure 6. Woman (52 years old) with cellulite appearance (a), and post-operational improvement of cellulite after 3 months (b).

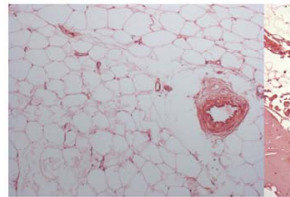

(a)

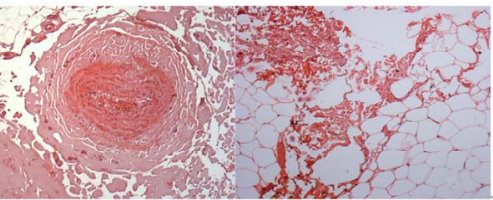

(b) (c)

Figure 7. Histological results of untreated skin (a), and immediately following RFAL treatment, showing coagulated vessels (b), and adipolysis (c).

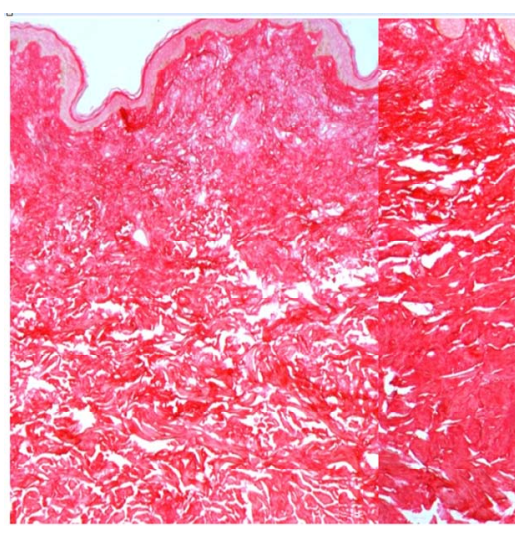

(a)

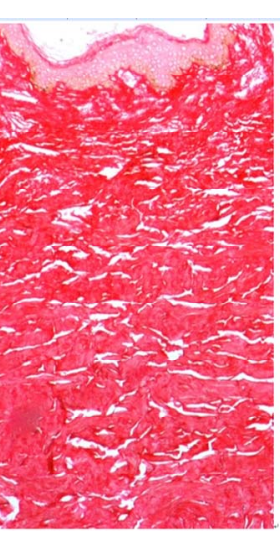

(b)

Figure 8. Histological results of untreated skin (a), and immediately following RFAL treatment, showing contraction of the skin with decreased space between collagen fibers (b).

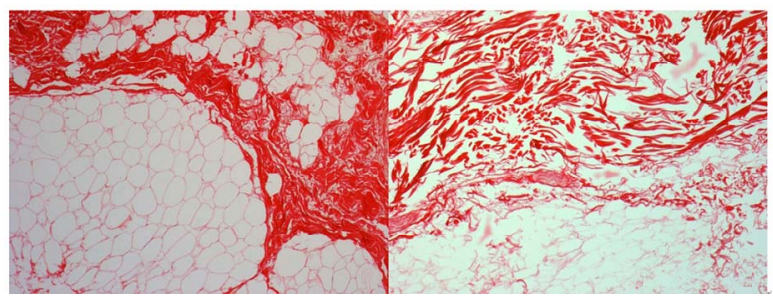

(a)

(b)

Figure 9. Histological results of untreated skin (a), and immediately following RFAL treatment, showing destruction of fibrous tracts in hypodermis and spacing of collagen fibers in deep dermis (b).
Long-term Results: skin samples from RFAL treated patients were maintained for 10 days in ex-vivo survival conditions according to GREDECO's technology, representing long-term results in-vivo. Histological quantification of dermal elastic and collagen fibers was evaluated by computerized image analysis at D10. The treated skin samples showed a statistically significant increase in their collagen levels, expressed as percentage of surface of analyzed dermis: $88.9 \% \pm 3.5 \%$ of collagen in the superficial-mid dermis versus $83.8 \% \pm 4.4 \%$ for untreated skin (6\% increase; $p=0.003)$. In deep dermis, treated samples showed a dense collagen band with $87 \%$ $\pm 6.0 \%$ versus $79.1 \% \pm 9.7 \%$ for control skin $(10 \%$ increase; $\mathrm{p}=0.04$ ). Formation of thick collagen band at the dermal-hypodermal junction may account for cellulite improvement (Figure 10).

Fragments from RFAL treated skin showed a statistically significant increase in its elastic fibers levels (9\%) in superficial-mid dermis in comparison with untreated skin $(7 \%)(28 \%$ increase; $p=0.003)$. Similar results were noted in the deep dermis with $7.5 \%$ versus $6 \%$ elastic fibers (25\% increase; $p=0.02$ ) (Figure 11). In deep dermis the mean length of an elastic fiber was statistically increased from 3.53 to $4.11 \mu \mathrm{m}$ in treated skin (16\% increase; $\mathrm{p}=0.04$ )

A significant increase of collagen synthesis was measured as $22.3 \mu \mathrm{g} / \mathrm{mg}$ skin biopsy in RFAL-treated samples

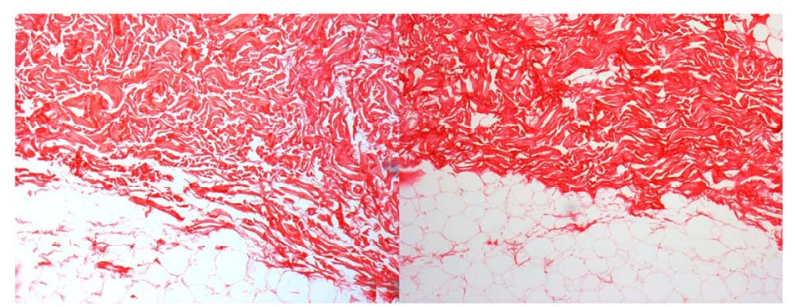

(a)

(b)

Figure 10. Histological results of untreated skin (a), and of RFAL-treated skin after 10 days in survival culture, showing thick collagen band at the dermal-hypodermal junction (b).

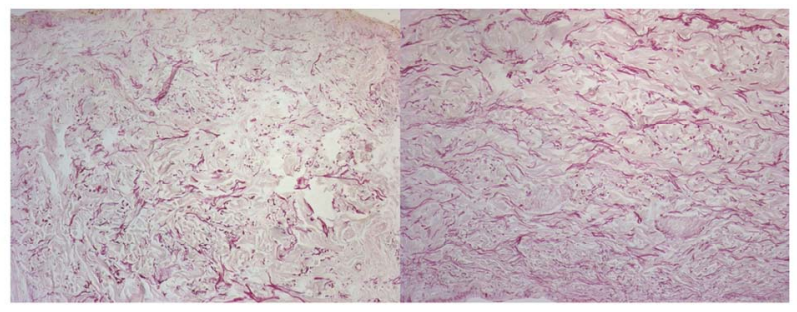

(a)

(b)

Figure 11. Histological results of untreated skin (a), and of RFAL-treated skin after 10 days in survival culture, showing increase of elastic fibers quantity (b). 
versus 19.1 in untreated skin (16.7\% increase; $p=0.03$ ).

A significant increase of elastin synthesis was also measured as $181 \mu \mathrm{g} / \mathrm{ml}$ supernatant in treated samples versus 146.8 in untreated skin $(23.3 \%$ increase; $\mathrm{p}=$ 0.045).

Average \% increase of dermal collagen and elastic fibers induced by RFAL after 10 days in culture are summarized in Table 1.

\section{Discussion}

Skin contraction and circumference reduction observed clinically after RFAL treatment, correlated with the histological findings by the GREDECO model applied in this study: a decrease of spaces between collagen fibers in superficial and mid dermis, increased dermal synthesis of collagen and elastin, as well as increased elastic fibers length. Coagulation of fat that may be effectively and moderately aspirated, and coagulation of blood vessels that is manifested in reduced ecchymosis, were also observed.

In addition, RFAL seems very promising in the treatment of cellulite, due to the formation of a thick collagen band at the dermal-hypodermal junction. This collagen reorganization may prevent the hernia of adipose tissue into the deep dermis, which is manifested as cellulite. The elimination of subcutaneous fat and the modulation of tissue fibers further contribute to cellulite improvement. This finding is of a particular interest in view of the inability of current liposuction technologies to improve cellulite; in fact, occasionally SAL is known to make cellulite worse.

Clinical results of circumference reduction and linear skin contraction of arms and abdomen showed amelioration between the 6th week and the 3rd month. Results stabilized after 3 months, indicating that healing of the underlying tightened tissues took place during the first 3 months post treatment. Results obtained after average healing of 3 months showed stability for at least 3 additional months. The cutaneous retraction is also tied to the coagulation of deep networks of hypodermal septa and fascias [16]. Further follow-up after one year, would substantiate the long term results.

The efficacy of skin tightening induced by RFAL is

Table 1. Long-term dermal fibers modification by RFAL.

\begin{tabular}{cc}
\hline Measured Parameter & \% Increase \\
\hline Collagen Level & 8.0 \\
Elastin level & 26.5 \\
Collagen Synthesis & 16.7 \\
Elastin Synthesis & 23.3 \\
Elastic fiber length & 16.0 \\
\hline
\end{tabular}

due to the dramatic changes of cutaneous retraction which were observed immediately after RF application. The higher the RF power, the higher and faster the temperature rise, inflicting more significant coagulation and thermal contraction within safety limits. This immediate shrinking is more pronounced in the abdomen (9\%) than in the arms (6\%), and is due to the introduction of more RF energy in abdomen than in arms.

The ex-vivo method of long-term skin culture used in this study [13] simulates the in-vivo environment. Moreover, the metabolic activity of skin fragments maintained under the GREDECO survival conditions is accelerated and represents a long-term in-vivo response [15]. All these results are due to the RF stimulation, as previously shown $[12,17,18]$. The final result is an improved body contour along with skin of improved quality and increased elasticity.

\section{Conclusions}

The RFAL technology is based on the delivery of direct RF energy into the subcutaneous fat to coagulate and softens adipose tissues for easy aspiration, and gently heat the subcutaneous and dermal fibrous matrix to sub-necrotic contractile levels. The heating is fast and uniform and when using appropriate settings and clinical monitoring of the target area, there are no adverse events. The RFAL technology involves three simultaneous clinical effects of coagulation, fat aspiration, and skin tightening.

RFAL technology offers superior performance compared with other methods of liposuction due to increased skin tightening and mild operation resulting in higher safety, patient tolerance, and fast recovery. In addition, the RFAL system is effective in cellulite treatment. As a result of the unique GREDECO methodology, it was possible to compare clinical and histological results, which were found to be highly correlated.

\section{REFERENCES}

[1] J. A. Klein, “The Tumescent Technique: Anaesthesia and Modifies Liposuction Technique,” Dermatologic Clinics, Vol. 8, No. 3, 1990, pp. 425-437.

[2] R. Graf, A. Auerswal, R. C. Damasio, R. Rippel, L. R. de Araujo, L. H. Bigarelli and C. L. Franck, "Ultrasound-Assisted Liposuction: An Analysis of 348 Cases," Aesthetic Plastic Surgery, Vol. 27, No. 2, 2003, pp. 146-153. doi:10.1007/s00266-002-1516-X

[3] C. Troilus, "Ultrasound Assisted Lipoplasty: Is It Really Safe?” Aesthetic Plastic Surgery, Vol. 23, No. 5, 1999, pp. 307-311. doi:10.1007/s002669900290

[4] S. S. Collawn, "Skin Tightening with Fractional Lasers, Radiofrequency, Smartlipo,” Annals of Plastic Surgery, 
Vol. 65, No. 5, 2010, pp. 526-529.

[5] B. D. Zelickson and T. D. Dressel, "Discussion of Laser Assisted Liposuccion," Lasers in Surgery and Medicine, Vol. 4, No. 10, 2009, pp. 709-713. doi:10.1002/lsm.20842

[6] G. Blugerman, D. Schavelzon and M. D. Paul, “A Safety and Feasibility Study of a Novel Radiofrequency-Assisted Liposuction Technology," Plastic and Reconstructive Surgery, Vol. 125, No. 3, 2010, pp. 998-1006. doi:10.1097/PRS.0b013e3181ce1820

[7] R. S. Mulholland, "An in-Depth Examination of Radiofrequency-Assisted Liposuction (RFAL)," Journal of Cosmetic Surgery \& Medicine, Vol. 4, No. 3, 2009, pp. 14-19.

[8] M. D. Paul, "Radiofrequency-Assisted Liposuction Comes of Age,” Plastic Surgery Practice, February 2009, pp. 18-19.

[9] M. Paul and R. S. Mulholland, "A New Approach for Adipose Tissue Treatment and Body Contouring Using Radiofrequency Assisted Liposuction," Aesthetic Plastic Surgery, Vol. 33, No. 5, 2009, pp. 687-694. doi:10.1007/s00266-009-9342-z

[10] B. M. Hantash, A. A. Ubeid, H. Chang, R. Kafi and B. Renton, "Bipolar Fractional Radiofrequency Treatment Induces Neoelasto-Genesis and Neocollagenesis,” Lasers in Surgery and Medicine, Vol. 41, No. 1, 2009, pp. 1-9. doi:10.1002/lsm.20731

[11] J. A. Klein, "Tumescent Technique for Local Anesthesia Improves Safety in Large-Volume Liposuction,” Plastic and Reconstructive Surgery, Vol. 92, No. 6, 1993, pp. 1099-1100. doi:10.1097/00006534-199311000-00015

[12] S. Boisnic, "Evaluation du Dispositif de Radiofréquence Tripolaire Regentm en Utilisant un Modèle Experimental de Peau Humaine,” Nouveau Journal de Chimie, Vol. 27, 2008, pp. 331-332.

[13] S. Boisnic, M. C. Branchet-Gumila, L. Benslama, Y. Le Charpentier and J. Arnaud-Battandier, "Long-Term Culture of Normal Skin to Test the Efficacy of a Hydroxy Acid-Containing Cream,” European Journal of Dermatology, Vol. 7, 1997, pp. 271-273.

[14] S. Boisnic, M. C. Branchet-Gumila, Y. Le Charpentier and C. Segard, "Repair of UVA-Induced Elastic Fiber and Collagen Damage by $0.05 \%$ Retinaldehyde Cream in an Ex-Vivo Human Skin Model,” Dermatology, Vol. 199, Supplement 1, 1999, pp. 43-48. doi:10.1159/000051378

[15] S. Boisnic, D. Licu, L. Ben Slama, M. C. Branhet-Gumila, H. Szpirglas and P. Dupuy, "Topical Retinaldehyde Treatment in Oral Lichen Planus and Leukoplakia,” International Journal of Tissue Reactions, Vol. 24, No. 4, 2002, pp. 123-130.

[16] M. Paul, G. Blugerman, M. Kreindel and R. S. Mulholland, "Three-Dimensional Radiofrequency Tissue Tightening: A Proposed Mechanism and Applications for Body Contouring," Annals of Plastic Surgery, Vol. 35, No. 1, 2010, pp. 87-95.

[17] S. Boisnic and M. C. Branchet, "Ex-Vivo Human Skin Evaluation of Localized Fat Reduction and Anti-Aging Effect by Tripollar Radio Fraquency Treatments,” Journal of Cosmetic and Laser Therapy, Vol. 12, No. 1, 2010, pp. 25-31. doi:10.3109/14764170903376232

[18] S. Boisnic, M. C. Branchet, O. Birnstiel and G. Beilin, "Clinical and Histopathological Study of the Tripollar Home-Use System for Body Treatments," European Journal of Dermatology, Vol. 20, No. 3, 2010, pp. 1-6. 